\title{
Coupling Identical one-dimensional Many-Body Localized Systems
}

\author{
Pranjal Bordia, ${ }^{1,2}$ Henrik P. Lüschen, ${ }^{1,2}$ Sean S. Hodgman, ${ }^{1,2,3}$ Michael Schreiber, ${ }^{1,2}$ \\ Immanuel Bloch, ${ }^{1,2}$ and Ulrich Schneider ${ }^{1,2,4}$ \\ ${ }^{1}$ Fakultät für Physik, Ludwig-Maximillians-Universität München, Schellingstraße 4, 80799 Munich, Germany \\ ${ }^{2}$ Max-Planck-Institut für Quantenoptik, Hans-Kopfermann-Straße 1, 85748 Garching, Germany \\ ${ }^{3}$ Research School of Physics and Engineering, Australian National University, Canberra ACT 0200, Australia \\ ${ }^{4}$ Cavendish Laboratory, University of Cambridge, J. J. Thomson Avenue, Cambridge CB3 OHE, United Kingdom
}

(Received 7 September 2015; published 4 April 2016)

\begin{abstract}
We experimentally study the effects of coupling one-dimensional many-body localized systems with identical disorder. Using a gas of ultracold fermions in an optical lattice, we artificially prepare an initial charge density wave in an array of $1 \mathrm{D}$ tubes with quasirandom on-site disorder and monitor the subsequent dynamics over several thousand tunneling times. We find a strikingly different behavior between manybody localization and Anderson localization. While the noninteracting Anderson case remains localized, in the interacting case any coupling between the tubes leads to a delocalization of the entire system.
\end{abstract}

DOI: 10.1103/PhysRevLett.116.140401

Introduction.-Many-body localization (MBL) marks a new paradigm in condensed matter and statistical physics. It describes an insulating phase in which a disordered, interacting many-body quantum system fails to act as its own heat bath [1-5]. In isolation, these systems will never achieve local thermal equilibrium and conventional statistical physics approaches break down. Unlike other insulating phases, MBL is not limited to ground states, but can even occur in all exited states of a disordered manybody system [6-10]. A dynamical phase transition separates the MBL phase from conventional ergodic phases $[11,12]$, in which the isolated system thermalizes. In these ergodic phases, any initial quantum information becomes rapidly diluted in the exponentially large Hilbert space, leading to decoherence. In contrast, in the localized insulating phase quantum information can persist locally for an infinite amount of time [5]. This could potentially render quantum information devices less susceptible to noise and disorder. For many decades it remained unclear whether such a localized phase could persist in a manybody system beyond the noninteracting limit of an Anderson insulator [13]. Today, both theory and experiment have shown evidence for the existence of an MBL phase in interacting 1D systems [5-7,14-16]. Nonetheless, many fundamental questions regarding this phase and the associated phase transition as well as its extension to higher dimensions [17] remain open, making it a highly active topic of current theoretical and experimental research.

One crucial requirement for the existence of a MBL phase is that no coupling to any external heat bath or bathlike structure exists. Any such coupling will eventually thermalize the system and ultimately destroy the nonthermalizing MBL phase [5,18-22]. Since any experimental system will inevitably be coupled-albeit potentially very weakly - to an environment, it is of critical importance to quantitatively understand the effect of such a coupling. Furthermore, studying the effects of weak couplings can help to experimentally identify an MBL phase and distinguish it from glasses or noninteracting Anderson localized phases.

Ultracold quantum gases in optical lattices form an ideal system to investigate these questions, as they are almost perfectly isolated from the external world and are highly controllable. Earlier experiments have investigated the interplay between disorder and interactions in the ground state of an isolated system of ultracold bosons in optical lattices [23-25] and studied the influence of disorder on transport properties for lattice fermions [26]. Recently, we were able to show that for a wide range of energy densities and interactions, an MBL phase exists in 1D Hubbard-type chains with quasirandom disorder [14].

In this work, we experimentally study the effects of coupling identically disordered 1D MBL systems to each other. In particular, the disordered Hamiltonian is identical for all 1D tubes, but the initial configuration of atoms differs between the tubes (Fig. 1). We find that the coupled systems can collectively serve as a bath for each other; i.e., coupling localized systems can result in delocalizing all of them.

Experiment.-Our experiments start with a two component Fermi gas of ${ }^{40} \mathrm{~K}$ atoms in an equal mixture of the two lowest hyperfine states $\left|F, m_{F}\right\rangle=\left|\frac{9}{2},-\frac{9}{2}\right\rangle \equiv|\downarrow\rangle$ and $\left|\frac{9}{2},-\frac{7}{2}\right\rangle \equiv|\uparrow\rangle$ with a total atom number of about $110-150 \times 10^{3}$ atoms. In the initial dipole trap, the atoms are at a temperature of $0.19(2) T_{F}$, where $T_{F}$ is the Fermi temperature. We load the Fermi gas into the lowest band of a deep, three-dimensional simple cubic optical lattice, where tunneling can be neglected. Along the longitudinal (x) direction, we then add a second (short) lattice (wavelength $\lambda_{s}=532 \mathrm{~nm}$ ) to the initial (long) lattice 


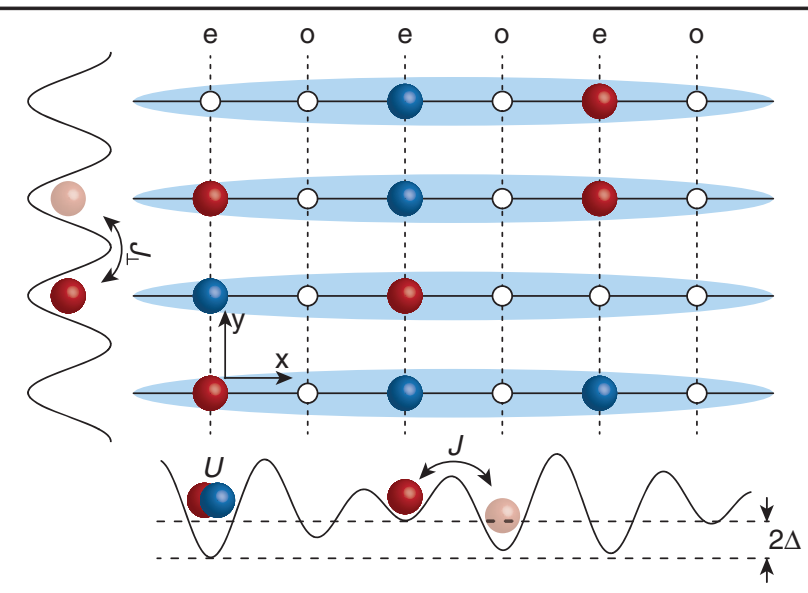

FIG. 1. Coupling identical MBL systems: A charge density wave (CDW) with atoms only occupying even sites $(e)$ is prepared in each of the identically disordered 1D tubes along the longitudinal $(x)$ direction, with hopping $J$, on-site interaction energy $U$, and disorder strength $\Delta$. Red and blue spheres indicate a typical distribution of $|\uparrow\rangle$ and $|\downarrow\rangle$ atoms. We monitor the time evolution of such a state for different intertube coupling strengths $J_{\perp}$, that is, different hopping amplitudes along the transverse $(y)$ direction.

$\left(\lambda_{l}=1064 \mathrm{~nm}\right)$. By controlling the phase of the short lattice during loading, we prepare a period-two "charge-density wave" (CDW), where only even sites are occupied in the 20 $E_{r}$ deep short lattice. Here, the recoil energy is denoted by $E_{r}=h^{2} / 2 m \lambda^{2}$, where $h$ is Planck's constant, $\lambda$ is the respective lattice wavelength, and $m$ is the atomic mass. The orthogonal lattices along $y$ and $z$ with a wavelength of $\lambda_{\perp}=738 \mathrm{~nm}$ are initially ramped up to $45 E_{r}$, creating an array of (almost) isolated 1D tubes. During lattice loading the interactions are kept strongly repulsive at a scattering length of $a=142 a_{0}$, where $a_{0}$ is the Bohr radius, by employing a Feshbach resonance centered at $202.1 \mathrm{G}$ [27]. This results in a doublon fraction, that is, the fraction of atoms on doubly occupied lattice sites, of $\leq 10 \%$.

After the preparation of the CDW in the deep lattices, the desired interactions for the ensuing evolution are set. Additionally, an incommensurate lattice of wavelength $\lambda_{d}=738 \mathrm{~nm}$ is superimposed along the $x$ direction to create quasirandom on-site disorder along the longitudinal direction. The system size is approximately 200 sites in the longitudinal and 120 sites in the transverse direction, and the central longitudinal tubes contains about 90 atoms [28]. After this preparation, the long lattice is quickly ramped to zero, the transverse $y$ lattice is ramped to its final value, which controls the transverse coupling $J_{\perp}$, and the short lattice is reduced to $8 E_{r}$. This last ramp enables tunneling along the tube and thereby initiates the dynamics. After a variable evolution time, we extract the imbalance between atoms on even and odd sites $\mathcal{I}=\left(N_{e}-N_{o}\right) /\left(N_{e}+N_{o}\right)$ [14]. Here, $N_{e}$ and $N_{o}$ denote the population of even and odd sites along the longitudinal direction and are extracted by mapping them to different bands of the superlattice [29].
The imbalance provides a measure of ergodicity breaking: It quickly decays to zero under any ergodic dynamics and any nonzero imbalance persisting at long times signifies a memory of the initial state and directly indicates localization [14,22,32].

Model.-Our system consists of an array of 1D tubes with identical quasirandom on-site disorder along the longitudinal direction. Each tube can be described by the Aubry-André model [33] with interactions [34], as depicted in Fig. 1. A finite hopping amplitude $J_{\perp}$ along the transverse $(y)$ direction introduces a coupling between adjacent tubes. The Hamiltonian of the system is given by

$$
\begin{aligned}
\hat{H}= & -J \sum_{i, j, \sigma}\left(\hat{c}_{i+1, j, \sigma}^{\dagger} \hat{c}_{i, j, \sigma}+\text { H.c. }\right) \\
& -J_{\perp} \sum_{i, j, \sigma}\left(\hat{c}_{i, j+1, \sigma}^{\dagger} \hat{c}_{i, j, \sigma}+\text { H.c. }\right) \\
& +\Delta \sum_{i, j, \sigma} \cos (2 \pi \beta i+\phi) \hat{n}_{i, j, \sigma}+U \sum_{i, j} \hat{n}_{i, j, \uparrow} \hat{n}_{i, j, \downarrow},
\end{aligned}
$$

where $J \approx h \times 500 \mathrm{~Hz}$ is the tunneling matrix element between neighboring sites along a tube and $J_{\perp}$ denotes the transverse $(y)$ hopping between the tubes. The creation (annihilation) operator for a fermion in spin state $\sigma \in$ $\{\uparrow, \downarrow\}$ on site $i$ in tube $j$ is $\hat{c}_{i, j, \sigma}^{\dagger}\left(\hat{c}_{i, j, \sigma}\right)$ and the local number operator is given by $\hat{n}_{i, j, \sigma}=\hat{c}_{i, j, \sigma}^{\dagger} \hat{c}_{i, j, \sigma}$. The quasirandom on-site disorder is characterized by the disorder amplitude $\Delta$, the incommensurable wavelength ratio $\beta=\lambda_{s} / \lambda_{d}$, and the relative phase $\phi$. Finally, the on-site interaction energy is given by $U$.

In the limit $J_{\perp} \rightarrow 0$, the system decouples into many one-dimensional tubes, which show many-body localization [14]. For our experiment, the accessible limits of almost zero intertube coupling $J_{\perp} \lesssim 10^{-3} J$ and equal coupling $J_{\perp}=J$ are termed the $1 \mathrm{D}^{+}$and $2 \mathrm{D}$ cases, respectively. Note that the experimentally achievable limit of the $1 \mathrm{D}^{+}$case is not the same as the ideal theoretical 1D case. A small but nonzero coupling remains for any finite transverse lattice depth and can always affect the dynamics at very long time scales.

Results.-We monitor the time evolution of the imbalance at disorder amplitude $\Delta=5 \mathrm{~J}$ for various interaction strengths $U$ in both the $1 \mathrm{D}^{+}$and $2 \mathrm{D}$ cases. This disorder strength is deep in the MBL regime for isolated 1D tubes. Figure 2 shows exemplary time traces at $U=0$ and $U=-6 J$, with all times given in units of the longitudinal tunneling time $\tau=\hbar / J$. We start with an out-ofequilibrium density wave with an initial imbalance of $\mathcal{I}(t=0)=0.91 \pm 0.03$ and observe a fast initial decrease of the imbalance up to approximately one tunneling time. This decrease is similar in all cases and corresponds to an initial relaxation in the longitudinal direction.

As shown in Fig. 2, in both noninteracting cases, the initial decrease is followed by highly damped oscillations 


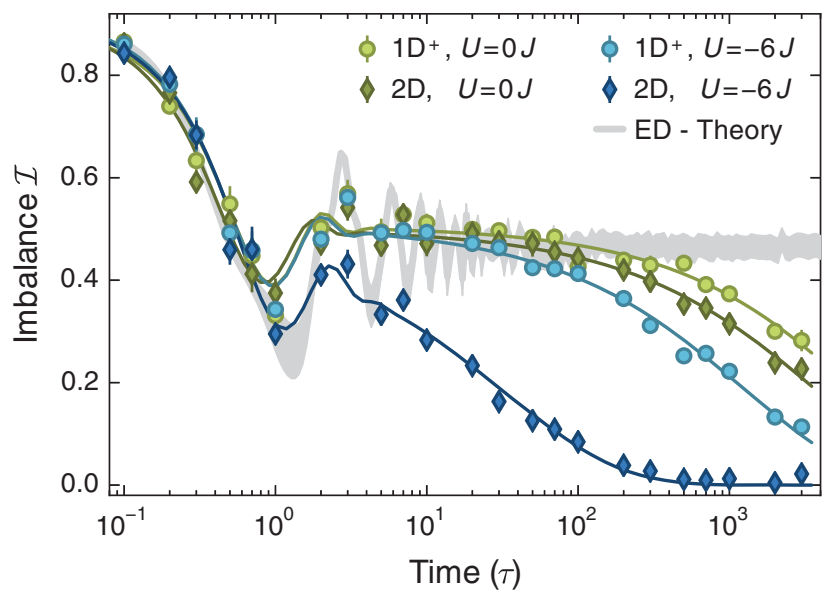

FIG. 2. Time evolution of a charge-density wave (CDW): An initially prepared 1D CDW evolves in a disordered system with disorder strength $\Delta=5 \mathrm{~J}$ along one axis. We measure the imbalance $\mathcal{I}$ after a given evolution time in $1 \mathrm{D}^{+}$(circles), where $J_{\perp} \lesssim 10^{-3} J$ and in $2 \mathrm{D}$ (diamonds), where $J_{\perp}=J$. Each data point is the average of 6 disorder phase realizations, with error bars showing the standard error of the mean. Solid lines are fits [28] from which we extract the imbalance lifetimes. Shown in gray is an exact diagonalization (ED) calculation for $J_{\perp}=0$, $\Delta=5 J$, and $U=0[28]$.

around a plateau at finite imbalance, closely matching the expected steady state for this Anderson localized system $[14,28]$. At very long times $(\gg 100 \tau)$, the curves start to deviate from this plateau and exhibit a slow decay. The corresponding lifetime is extracted by fitting the imbalance traces to a damped sinusoid, which models the initial fast relaxation, multiplied by a stretched exponential to capture the slow decay [28]. This stretched exponential is of the form $e^{-(\Gamma t)^{\beta}}$, where $t$ is the evolution time, $\Gamma$ is the decay rate, and $\beta$ is the stretching exponent. This model fits all time traces consistently better than a simple exponential decay and has also been observed in glasses, disordered materials, and polymers [35-37]. We define our imbalance lifetime $T_{1 / e}=1 / \Gamma$.

In the absence of interactions, the observed long time dynamics is dominated by classical noise, photon scattering from lattice beams, and other technical imperfections. These processes couple the system to the environment and over time delocalize it [38,39]. In addition, these experimental imperfections also give rise to an atom number decay, which limits the lifetime of atoms in the lattice to $0.5-1.1 \times 10^{4} \tau$ [28]. In the absence of interactions, the observed dynamics in $1 \mathrm{D}^{+}$and $2 \mathrm{D}$ are almost the same, since the disorder potential is identical in all tubes [28]. Therefore, the 2D system is separable, and the longitudinal and transverse directions are decoupled. Note that we do not expect this to hold if the disorder were different in different tubes [40]. Adding interactions, however, breaks the separability of the system and the transverse dynamics can now affect the imbalance along the longitudinal direction. Since there is no disorder along the transverse direction, particles are free to move along this direction. In the interacting case, this couples the originally localized tubes such that they collectively act as a bath for each other and thereby delocalize the entire system. As a consequence, the interacting 2D trace in Fig. 2 displays no plateau but instead shows a fast decay. To understand this behavior further, we map out the imbalance lifetimes in the $1 \mathrm{D}^{+}$and $2 \mathrm{D}$ cases for various interaction strengths (Fig. 3). We find that in 2D, even small interactions are sufficient to dramatically reduce the imbalance lifetime to less than $100 \tau$.

Although MBL is expected to be stable in the isolated 1D case, the $1 \mathrm{D}^{+}$data in Fig. 3 show qualitatively similar behavior to the $2 \mathrm{D}$ case, but with a much weaker decrease of imbalance lifetimes with increasing interactions. This suggests that the small but nonzero intertube coupling also limits the lifetime in the $1 \mathrm{D}^{+}$case. The gray shaded region in Fig. 3 marks the range of observed atom number lifetimes, which approximately coincides with the range of noninteracting imbalance lifetimes, indicating the relevance of technical imperfections on this time scale.

$1 D-2 D$ crossover. - In order to directly test the effect of residual couplings, we vary the strength of the intertube coupling between the $2 \mathrm{D}$ and the $1 \mathrm{D}^{+}$limits for four different interactions (Fig. 4). We observe increasing lifetimes for decreasing coupling strengths $J_{\perp}$ in all interacting cases. For small but finite coupling strengths, we observe a linear trend on a log-log scale (Fig. 4), suggesting a power-law dependence. For strong intertube coupling $\left(J_{\perp} / J \gtrsim 0.1\right)$, there is a crossover to a faster decay. The fitted exponents are surprisingly small $(|k|<1)$ and depend nonmonotonically on the interactions. In the noninteracting

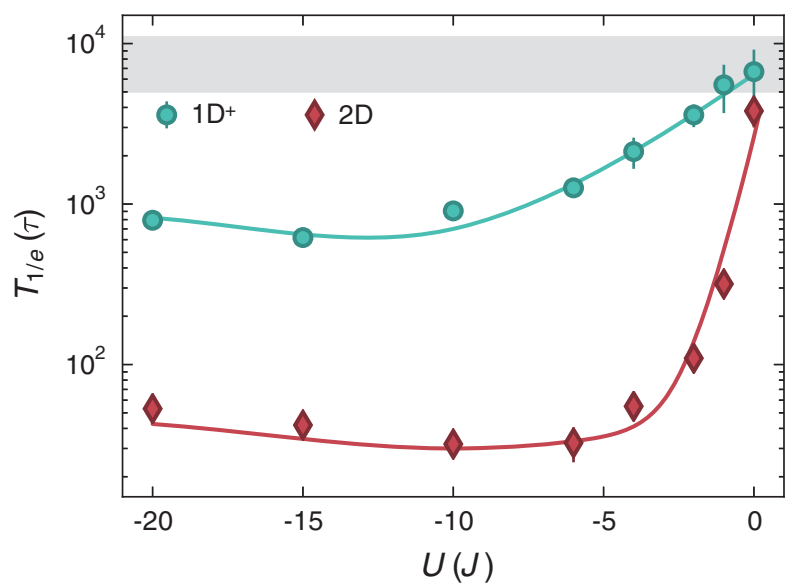

FIG. 3. Imbalance lifetimes vs interactions $(U)$ : Imbalance lifetimes at $\Delta=5 \mathrm{~J}$ for $1 \mathrm{D}^{+}$and $2 \mathrm{D}$. We note that the $1 \mathrm{D}^{+}$ case differs crucially from the ideal isolated 1D case. The lifetimes were extracted from fits to time traces such as in Fig. 2. Error bars denote fit uncertainty [28]. The gray shaded area indicates the range of measured atom number lifetimes, while the lines are guides to the eye. 


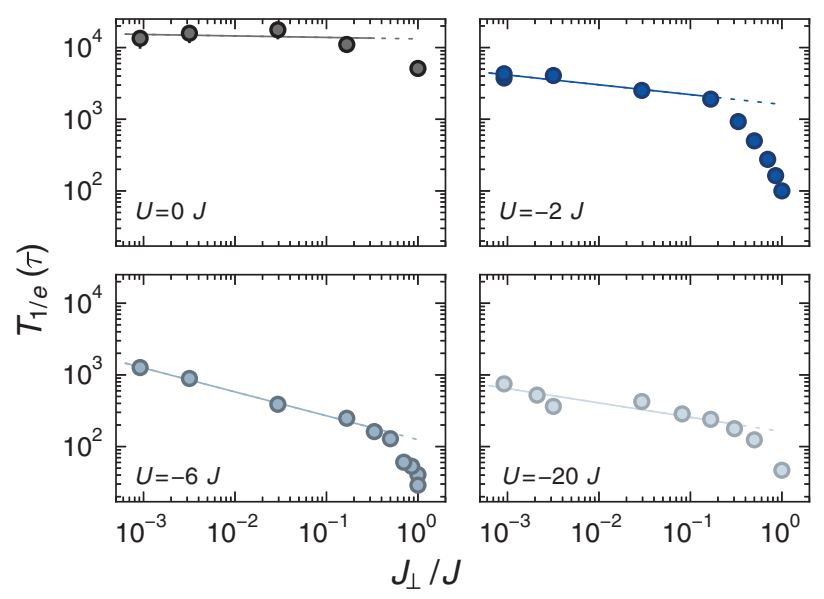

FIG. 4. Imbalance lifetimes in the 1D-2D crossover: The coupling strength $J_{\perp}$ between adjacent tubes is varied continuously for $\Delta=5 \mathrm{~J}$ at four different interactions $U$. Here, $J_{\perp} / J \lesssim$ $10^{-3}$ and $J_{\perp} / J=10^{0}$ correspond to the $1 \mathrm{D}^{+}$and the $2 \mathrm{D}$ cases, respectively. The lack of saturation as $J_{\perp} \rightarrow 0$ indicates that the residual intertube coupling still limits the imbalance lifetime in the $1 \mathrm{D}^{+}$case. Solid lines denote power-law fits $\propto J_{\perp}^{k}$ for small $J_{\perp}$, with fitted exponents $k(U / J)$ of $k(0)=0.00(4), k(-2)=$ $-0.09(2), k(-6)=-0.30(1)$, and $k(-20)=-0.16(3)$. We note that in principle the tunneling along the $z$ direction becomes sizable for the smallest $J_{\perp}$, as $J_{\perp}^{z} / J \sim 10^{-3}$.

limit $U \rightarrow 0$, the lifetimes are on the order of the atom number lifetime and become independent of the transverse coupling due to the separability of the problem. This highlights the striking difference between MBL and Anderson localization.

Extrapolating towards the limit of the true 1D case $\left(J_{\perp} \rightarrow 0\right)$, we would expect the lifetimes of the interacting system to saturate once the intertube coupling is no longer the dominant decay mechanism. However, in the experimentally accessible regime we cannot observe any signs of saturation, strongly suggesting that the interacting lifetimes are still limited by the nonzero residual transverse coupling to the neighboring tubes, even in the $1 \mathrm{D}^{+}$case.

Constant evolution time.-The interaction dependence is also visible in the imbalance value measured after a fixed evolution time of around $40 \tau$, as shown in Fig. 5. We observe a substantial decrease of the 2D imbalance with small interactions, which is consistent with the sharply decreasing lifetimes. Additionally, Fig. 5 shows that the dynamics are symmetric around $U=0$, which is expected due to a dynamical symmetry of the Fermi-Hubbard model [30]. Interestingly, we observe an increasing imbalance in the $2 \mathrm{D}$ data for strong interactions. We checked that this increase is not due to doublons by removing any residual doublons with a pulse of near-resonant light [41] prior to the evolution. This increase in lifetime might be due to the reduced phase space for scattering in the hard-core limit $(U \gg J)$. A recent theoretical study using a cluster

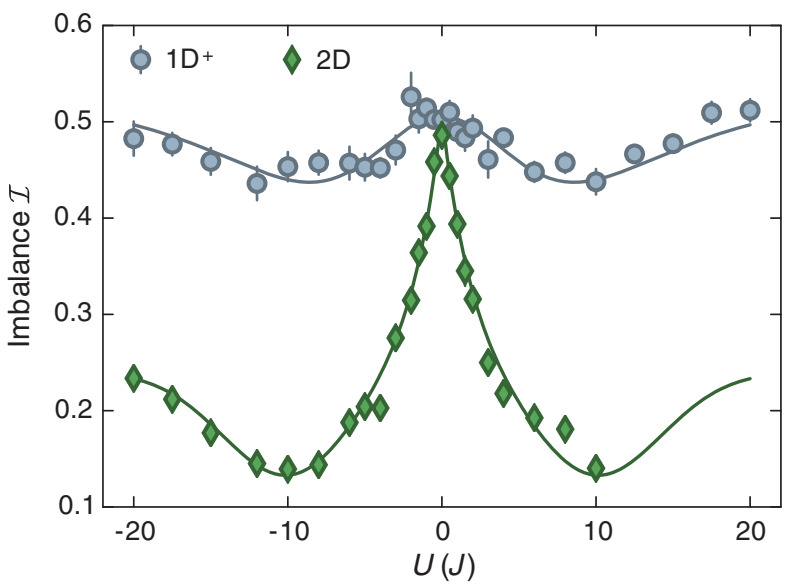

FIG. 5. Imbalance vs interactions $(U)$ at fixed times: Data taken at $\Delta=5 \mathrm{~J}$ averaged over three different times $(38 \tau-41 \tau)$ and 4 disorder phases $\phi$. In the $1 \mathrm{D}^{+}$case (circles), the imbalance corresponds to the stationary value [14], whereas in the 2D case (diamonds) it is indicative of the decay lifetime. Solid lines are guides to the eye. The $2 \mathrm{D}$ case is limited to $U / J \leq 10$ due to the details of the used Feshbach resonance [27].

expansion method on a smaller system $(8 \times 8$ sites $)$ observed a similar trend [42].

At $40 \tau$, the $1 \mathrm{D}^{+}$case is dominated by the $1 \mathrm{D}$ plateau value and shows the characteristic $W$ shape of the steady state imbalance of the MBL system [14], with little influence from the (much longer) lifetimes. As shown in the Supplemental Material [28], we additionally find that the imbalance lifetime in all cases increases strongly for larger disorder.

Conclusion.-We have studied the stability of manybody localized 1D systems under an intertube coupling. We found that even weak couplings have a delocalizing effect on the MBL phase, while leaving the noninteracting Anderson limit unchanged. This highlights the differences between these two regimes and shows the principal fragility of MBL with respect to coupling to any external heat bath [5,18-21]. Furthermore, we have not observed any saturation in imbalance lifetimes even for the smallest couplings we could attain, indicating that this intertube coupling is the dominant decay mechanism in this MBL experiment. Nonetheless, for strong disorders the achieved lifetimes already exceed the lifetimes of typical many-body states, such as, e.g., superfluid states in optical lattices. This demonstrates the stability of MBL with respect to other experimental imperfections and increases the prospects of realizing localization protected order [43-45] and applications in quantum information [5].

An important next step will be to extend this study to the "true" 2D case with disorder along both directions. In addition, future experiments should also be able to address the question of the stability of MBL under external influences, such as photon scattering $[38,46]$ and time-dependent modulations $[47,48]$. Finally, it would 
also be interesting to search for MBL in bosonic systems [49] and using other observables such as the growth of entanglement entropy and response to generalized interferometric probes [50] when a bath is added to an otherwise perfectly MBL system [22].

We acknowledge useful discussions with E. Altman, E. Demler, M. Fischer, D. Huse, M. Knap, R. Nandkishore and A. Pal. We acknowledge financial support by the European Commision (UQUAM, AQuS) and the Nanosystems Initiative Munich (NIM).

[1] J. M. Deutsch, Quantum statistical mechanics in a closed system, Phys. Rev. A 43, 2046 (1991).

[2] M. Srednicki, Chaos and quantum thermalization, Phys. Rev. E 50, 888 (1994).

[3] M. Rigol, V. Dunjko, and M. Olshanii, Thermalization and its mechanism for generic isolated quantum systems, Nature (London) 452, 854 (2008).

[4] A. Polkovnikov, K. Sengupta, A. Silva, and M. Vengalattore, Colloquium: Nonequilibrium dynamics of closed interacting quantum systems, Rev. Mod. Phys. 83, 863 (2011).

[5] R. Nandkishore and D. A. Huse, Many-body localization and thermalization in quantum statistical mechanics, Annu. Rev. Condens. Matter Phys. 6, 15 (2015).

[6] D. M. Basko, I. L. Aleiner, and B. L. Altschuler, Metal-insulator transition in a weakly interacting manyelectron system with localized single-particle states, Ann. Phys. (Amsterdam) 321, 1126 (2006).

[7] I. V. Gornyi, A. D. Mirlin, and D. G. Polyakov, Interacting Electrons in Disordered Wires: Anderson Localization and Low-T Transport, Phys. Rev. Lett. 95, 206603 (2005).

[8] V. Oganesyan and D. A. Huse, Localization of interacting fermions at high temperature, Phys. Rev. B 75, 155111 (2007).

[9] J. Z. Imbrie, On many-body localization for quantum spin chains, arXiv:1403.7837.

[10] V. P. Michal, I. L. Aleiner, B. L. Altshuler, and G. V. Shlyapnikov, Finite-temperature fluid-insulator transition of strongly interacting 1D disordered bosons, arXiv: 1502.00282 .

[11] A. Pal and D. A. Huse, Many-body localization phase transition, Phys. Rev. B 82, 174411 (2010).

[12] R. Vosk and E. Altman, Many-Body Localization in One Dimension as a Dynamical Renormalization Group Fixed Point, Phys. Rev. Lett. 110, 067204 (2013).

[13] P. W. Anderson, Absence of diffusion in certain random lattices, Phys. Rev. 109, 1492 (1958).

[14] M. Schreiber, S. S. Hodgman, P. Bordia, H. P. Lüschen, M. H. Fischer, R. Vosk, E. Altman, U. Schneider, and I. Bloch, Observation of many-body localization of interacting fermions in a quasirandom optical lattice, Science 349, 842 (2015).

[15] E. Altman and R. Vosk, Universal dynamics and renormalization in many-body-localized systems, Annu. Rev. Condens. Matter Phys. 6, 383 (2015).
[16] J. Smith, A. Lee, P. Richerme, B. Neyenhuis, P. W. Hess, P. Hauke, M. Heyl, D. A. Huse, and C. Monroe, Many-body localization in a quantum simulator with programmable random disorder, arXiv:1508.07026.

[17] N. Y. Yao, C. R. Laumann, S. Gopalakrishnan, M. Knap, M. Müller, E. A. Demler, and M. D. Lukin, Many-Body Localization in Dipolar Systems, Phys. Rev. Lett. 113, 243002 (2014).

[18] N. F. Mott, Conduction in non-crystalline materials, Philos. Mag. 19, 835 (1969).

[19] R. Nandkishore, S. Gopalakrishnan, and D. A. Huse, Spectral features of a many-body-localized system weakly coupled to a bath, Phys. Rev. B 90, 064203 (2014).

[20] S. Johri, R. Nandkishore, and R. N. Bhatt, Many-Body Localization in Imperfectly Isolated Quantum Systems, Phys. Rev. Lett. 114, 117401 (2015).

[21] D. A. Huse, R. Nandkishore, F. Pietracaprina, V. Ros, and A. Scardicchio, Localized systems coupled to small baths: From Anderson to Zeno, Phys. Rev. B 92, 014203 (2015).

[22] E. Levi, M. Heyl, I. Lesanovsky, and J. P. Garrahan, What survives of many-body localization in the presence of dissipation, arXiv:1510.04634.

[23] B. Deissler, M. Zaccanti, G. Roati, C. DErrico, M. Fattori, M. Modugno, G. Modugno, and M. Inguscio, Delocalization of a disordered bosonic system by repulsive interactions, Nat. Phys. 6, 354 (2010).

[24] B. Gadway, D. Pertot, J. Reeves, M. Vogt, and D. Schneble, Glassy Behavior in a Binary Atomic Mixture, Phys. Rev. Lett. 107, 145306 (2011).

[25] C. D’Errico, E. Lucioni, L. Tanzi, L. Gori, G. Roux, I. P. McCulloch, T. Giamarchi, M. Inguscio, and G. Modugno, Observation of a Disordered Bosonic Insulator from Weak to Strong Interactions, Phys. Rev. Lett. 113, 095301 (2014).

[26] S. S. Kondov, W. R. McGehee, W. Xu, and B. DeMarco, Disorder-Induced Localization in a Strongly Correlated Atomic Hubbard Gas, Phys. Rev. Lett. 114, 083002 (2015).

[27] C. A. Regal, C. Ticknor, J. L. Bohn, and D. S. Jin, Creation of ultracold molecules from a fermi gas of atoms, Nature (London) 424, 47 (2003).

[28] See Supplemental Material at http://link.aps.org/ supplemental/10.1103/PhysRevLett.116.140401, which includes Refs. [14,27,29-31], for details of the experimental procedures along with supporting material.

[29] S. Trotzky, Y.-A. Chen, A. Flesch, I. P. McCulloch, U. Schollwöck, J. Eisert, and I. Bloch, Probing the relaxation towards equilibrium in an isolated strongly correlated onedimensional Bose gas, Nat. Phys. 8, 325 (2012).

[30] U. Schneider, L. Hackermüller, J. P. Ronzheimer, S. Will, S. Braun, T. Best, I. Bloch, E. Demler, S. Mandt, D. Rasch, and A. Rosch, Fermionic transport and out-of-equilibrium dynamics in a homogeneous Hubbard model with ultracold atoms, Nat. Phys. 8, 213 (2012).

[31] U. Schneider, L. Hackermüller, S. Will, Th. Best, I. Bloch, T. A. Costi, R. W. Helmes, D. Rasch, and A. Rosch, Metallic and insulating phases of repulsively interacting fermions in a 3D optical lattice, Science 322, 1520 (2008). 
[32] R. Mondaini and M. Rigol, Many-body localization and thermalization in disordered Hubbard chains, Phys. Rev. A 92, 041601 (2015).

[33] S. Aubry and G. André, Analyticity breaking and Anderson localization in incommensurate lattices, Ann. Israel Phys. Soc. 3, 133 (1980).

[34] S. Iyer, V. Oganesyan, G. Refael, and D. A. Huse, Manybody localization in a quasiperiodic system, Phys. Rev. B 87, 134202 (2013).

[35] R. V. Chamberlin, G. Mozurkewich, and R. Orbach, Time Decay of the Remanent Magnetization in Spin-Glasses, Phys. Rev. Lett. 52, 867 (1984).

[36] U. Even, K. Rademann, J. Jortner, N. Manor, and R. Reisfeld, Electronic Energy Transfer on Fractals, Phys. Rev. Lett. 52, 2164 (1984).

[37] J.C. Phillips, Stretched exponential relaxation in molecular and electronic glasses, Rep. Prog. Phys. 59, 1133 (1996).

[38] S. A. Gurvitz, Delocalization in the Anderson Model due to a Local Measurement, Phys. Rev. Lett. 85, 812 (2000).

[39] B. Nowak, J. J. Kinnunen, M. J. Holland, and P. Schlagheck, Delocalization of ultracold atoms in a disordered potential due to light scattering, Phys. Rev. A 86, 043610 (2012).

[40] E. Abrahams, P. W. Anderson, D. C. Licciardello, and T. V. Ramakrishnan, Scaling Theory of Localization: Absence of Quantum Diffusion in Two Dimensions, Phys. Rev. Lett. 42, 673 (1979).

[41] J. P. Ronzheimer, M. Schreiber, S. Braun, S. S. Hodgman, S. Langer, I. P. McCulloch, F. Heidrich-Meisner, I. Bloch, and U. Schneider, Expansion Dynamics of Interacting Bosons in Homogeneous Lattices in One and Two Dimensions, Phys. Rev. Lett. 110, 205301 (2013).
[42] M. D. Reichl and E. J. Mueller, Dynamics of pattern-loaded fermions in bichromatic optical lattices, arXiv:1508.00472.

[43] D. A. Huse, R. Nandkishore, V. Oganesyan, A. Pal, and S. L. Sondhi, Localization-protected quantum order, Phys. Rev. B 88, 014206 (2013).

[44] B. Bauer and C. Nayak, Area laws in a many-body localized state and its implications for topological order, J. Stat. Mech. (2013) P09005.

[45] A. Chandran, V. Khemani, C. R. Laumann, and S. L. Sondhi, Many-body localization and symmetry-protected topological order, Phys. Rev. B 89, 144201 (2014).

[46] B. Nowak, J. J. Kinnunen, M. J. Holland, and P. Schlagheck, Delocalization of ultracold atoms in a disordered potential due to light scattering, Phys. Rev. A 86, 043610 (2012).

[47] S. Gopalakrishnan, M. Müller, V. Khemani, M. Knap, E. Demler, and D. A. Huse, Low-frequency conductivity in many-body localized systems, Phys. Rev. B 92, 104202 (2015).

[48] K. Agarwal, S. Gopalakrishnan, M. Knap, M. Müller, and E. Demler, Anomalous Diffusion and Griffiths Effects near the Many-Body Localization Transition, Phys. Rev. Lett. 114, 160401 (2015).

[49] I. L. Aleiner, B. L. Altshuler, and G. V. Shlyapnikov, A finite-temperature phase transition for disordered weakly interacting bosons in one dimension, Nat. Phys. 6, 900 (2010).

[50] M. Serbyn, M. Knap, S. Gopalakrishnan, Z. Papić, N. Y. Yao, C. R. Laumann, D. A. Abanin, M. D. Lukin, and E. A. Demler, Interferometric Probes of Many-Body Localization, Phys. Rev. Lett. 113, 147204 (2014). 